\title{
A comprehensive analysis of the factors of positive pelvic lymph nodes on survival of cervical cancer patients with 2018 FIGO stage IIICIP
}

This article was published in the following Dove Press journal: Cancer Management and Research

\author{
Ding-Ding Yan \\ Qiu Tang \\ Ye-Qiang Tu \\ Jian-Hong Chen \\ Xiao-Juan Lv
}

Department of Gynecologic Oncology, Zhejiang Cancer Hospital, Hangzhou, People's Republic of China
Correspondence: Qiu Tang

Department of Gynecologic Oncology,

Zhejiang Cancer Hospital, No. I, Guangji

Road, Bansan Bridge, Gongshu District,

Hangzhou 310022, People's Republic of China

Tel +86 57I 88I2 2042

Fax +8657188122508

Email tangqiu@zjcc.org.cn
Objective: To evaluate the factors associated with positive pelvic lymph nodes (LNs) on the survival of patients with 2018 FIGO stage IIIC1p cervical cancer.

Methods: We retrospectively analyzed 155 patients with pelvic lymph node metastasis (LNM) confirmed by pathology after radical resection of cervical cancer treated at Zhejiang Cancer Hospital, China, between March 2008 and October 2011. We analyzed the influence of the factors associated with positive pelvic LNs on the survival of patients.

Results: The 5-year progress-free survival (PFS) and overall survival (OS) of patients were $78.1 \%$ and $81.9 \%$, respectively. The 5-year PFS and OS of patients with more than 2 LNM were worse compared with patients with 1 or 2 LNM $(68.4 \%$ vs $83.7 \%, p=0.013 ; 72.4 \%$ vs $87.6 \%, p=0.017$, respectively). The 5 -year PFS and OS of patients with more than 2 LNM sites were worse than that of patients with 1 or 2 LNM sites $(60.0 \%$ vs $82.4 \%, p=0.008$; $70.0 \%$ vs $84.8 \%, p=0.045$, respectively). The 5 -year PFS and OS of patients with common iliac LNM was poorer than that of patients without common iliac LNM (60.7\% vs $81.9 \%$, $p=0.008 ; 67.9 \%$ vs $85.0 \%, p=0.020$, respectively). Compared with other patients, the survival of patients with these three factors (more than 2 LNM, more than 2 LNM sites, and common iliac LNM) was the worst $(p<0.05)$.

Conclusion: More than 2 LNM, more than 2 LNM sites, and common iliac LNM were predictive factors of poor survival in stage IIIC1p cervical cancer patients. Survival of patients with stage IIIC1p cervical cancer declined with increasing presence of such factors. The combined evaluation of the factors associated with positive pelvic LNs is a more comprehensive and pragmatic approach in evaluating the prognosis of cervical cancer.

Keywords: cervical cancer, metastasis, prognosis, lymph nodes

\section{Background}

The FIGO Committee revised the staging system of cervical cancer in 2018 . ${ }^{1}$ One of the main changes is that stage IB is now classified into the following substages according to tumor size: stage IB1 $(<2 \mathrm{~cm})$, stage IB2 $(2-3.9 \mathrm{~cm})$, and stage IB3 $(\geq 4 \mathrm{~cm})$. Another change is the addition of stage IIIC1 and stage IIIC2 considering the influence of LNM on prognosis. IIIC1 refers to pelvic pelvic lymph node metastasis (LNM) only and IIIC2 refers to para-aortic LNM. When pelvic lymph node metastasis is confirmed by pathologic findings, this is designated Stage IIIC1p. The revised FIGO staging system has received much attention. Matsuo et al reported that the revised IB stage was not only closely related to the survival of patients but also more effective in guiding the treatment of 
patients. ${ }^{2}$ However, there is some controversy regarding staging of IIIC1. Matsuo et al found that patients with stage IIIC1 had superior cervical cancer-specific survival compared with IIIA-IIIB disease. In addition, the survival of patients with stage IIIC1 declines with increasing T-stage, ${ }^{2}$ which indicates that there is heterogeneity in those with stage IIIC1. Several studies have shown that the different factors of pelvic metastatic lymph nodes were associated with the prognosis of cervical cancer patients. The higher the number of LNM and sites affected, the poorer the survival prospects. $^{3-7}$ Simultaneously, common iliac LNM is especially associated with poor survival. ${ }^{8-10}$ However, Matsuo's validation of the 2018 FIGO staging system did not analyze the impact of some factors associated with metastatic lymph nodes (LNs) on prognosis. The aim of our research was to analyze the effect of some factors associated with metastatic LNs in stage IIIC1p on survival and to verify the rationale behind the new staging designation of IIIC1p.

\section{Methods}

\section{Ethics statement}

This study was approved by the institutional ethical review board of the Zhejiang Cancer Hospital, Zhejiang, China. All procedures were performed in accordance with the tenets of the Helsinki Declaration. Written informed consent was obtained from patients.

\section{Patients}

We retrospectively analyzed 155 pelvic LNM cervical cancer patients with early stage disease who underwent primary surgical treatment in the Gynecologic Oncology Department of Zhejiang Cancer Hospital from March 2008 to October 2011. A flow chart of the study population is shown in Figure 1. Table 1 shows the characteristics of patients. The mean age was 45 years (34-66 years). All patients were treated for the first time and had not received radiotherapy or chemotherapy prior to surgery. All patients did not have secondary tumors. All 155 patients who were confirmed as having pelvic LNM by postoperative pathology were classified as stage IIIC1p according to the 2018 FIGO staging system. Of 155 patients, 28 (18.1\%) had affected common iliac LNs, 127 (91.9\%) had affected pelvic LNs other than common iliac LNs; $97(62.6 \%)$ had 1 or 2 positive pelvic LNs, and 58 (47.4\%) had more than 2 positive pelvic LNs; 125 (80.6\%) had 1 or 2 pelvic LNM sites affected, and $30(19.4 \%)$ had more than 2 pelvic LNM sites affected.

\section{Treatment}

\section{Surgery}

All patients underwent radical hysterectomy and systematic bilateral pelvic lymphadenectomy. If common iliac LNs were identified as being positive by the intraoperative frozen section or the para-aortic LNs were

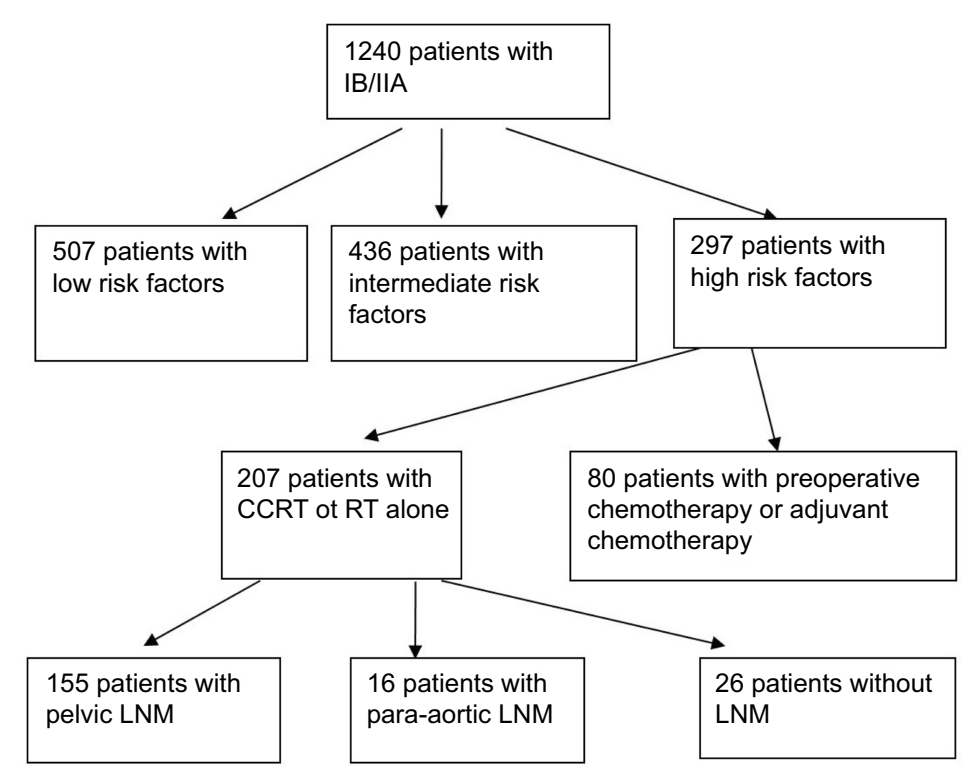

Figure I Flow chart of the study population.

Abbreviations: CCRT, concurrent chemoradiotherapy, RT, radiotherapy, LNM, lymph node metastasis. 
Table I Patient and disease characteristics

\begin{tabular}{|c|c|}
\hline Variable & $\mathbf{N}(\%)$ \\
\hline Median age (years) & $45(34-66)$ \\
\hline \multicolumn{2}{|l|}{ Pathologic type } \\
\hline Squamous & $135(87.1)$ \\
\hline adeno and adenosquamous & $20(12.9)$ \\
\hline \multicolumn{2}{|l|}{2009 FIGO } \\
\hline$|\mathrm{B}|$ & $67(43.2)$ \\
\hline IB2 & $30(19.4)$ \\
\hline$\| A \mid$ & $32(20.6)$ \\
\hline IIA2 & $26(16.8)$ \\
\hline \multicolumn{2}{|l|}{ Tumor size } \\
\hline $\mathrm{TI}(\leq 2 \mathrm{~cm})$ & $23(14.8)$ \\
\hline $\mathrm{T} 2(2<\mathrm{T} \leq 4 \mathrm{~cm})$ & $67(43.2)$ \\
\hline $\mathrm{T} 3(>4 \mathrm{~cm})$ & $65(41.9)$ \\
\hline \multicolumn{2}{|l|}{ DSI } \\
\hline$\leq 1 / 2$ & $23(14.8)$ \\
\hline$>1 / 2$ & $132(85.2)$ \\
\hline \multicolumn{2}{|l|}{ LVSI } \\
\hline Negative & $36(23.2)$ \\
\hline Positive & $119(76.8)$ \\
\hline \multicolumn{2}{|l|}{ Surgical margin } \\
\hline Negative & $148(95.5)$ \\
\hline Positive & $7(4.5)$ \\
\hline \multicolumn{2}{|l|}{$\mathrm{PI}$} \\
\hline Negative & $145(93.5)$ \\
\hline Positive & $10(6.5)$ \\
\hline \multicolumn{2}{|l|}{ Number of LNM } \\
\hline $1-2$ & $97(62.6)$ \\
\hline$\geq 3$ & $58(47.4)$ \\
\hline \multicolumn{2}{|l|}{ Site of PLN } \\
\hline Common iliac & $28(18.1)$ \\
\hline Common iliac unaffected & $127(81.9)$ \\
\hline \multicolumn{2}{|l|}{ Number of LNM sites } \\
\hline $\mathrm{I}-2$ & $125(80.6)$ \\
\hline$\geq 3$ & $30(19.4)$ \\
\hline \multicolumn{2}{|l|}{ Type of lymphadenectomy } \\
\hline Pelvic only & $113(72.9))$ \\
\hline Pelvic + para-aortic & $42(27.1)$ \\
\hline \multicolumn{2}{|l|}{ No. of resected nodes } \\
\hline$\leq 18$ & $46(29.7)$ \\
\hline$>18$ & $109(70.3)$ \\
\hline \multicolumn{2}{|l|}{ Therapy model } \\
\hline RT & $39(25.2)$ \\
\hline CCRT & $116(74.8)$ \\
\hline
\end{tabular}

Abbreviations: FIGO, International Federation of Gynecology and Obstetrics; DSI, depth of stromal invasion; LVSI, lymphovascular space invasion; PI, parametrial invasion; LNM, lymph node metastasis; PLN, pelvic lymph node; RT, radiotherapy; CCRT, concurrent radiotherapy and chemotherapy. identified as suspicious by visualization and palpation, para-aortic lymphadenectomy was also performed during radical surgeries.

\section{Radiotherapy}

External-beam radiation therapy to the pelvis was conducted on all patients. If common iliac LNs or paraaortic LNs were positive, extended-field radiation was given. The radiation dose was administered at 45-50.4 Gy in 25-28 fractions, 5 times per week. If patients had close or positive vaginal resection margins, a vaginal brachytherapy boost of approximately 20 Gy in 4 fractions was performed.

\section{Chemotherapy}

One hundred and sixteen (74.8\%) patients received concurrent chemotherapy during radiotherapy. Twenty-six patients were treated with weekly cisplatin chemotherapy (DDP group: daily $40 \mathrm{mg} / \mathrm{m}^{2}$ intravenously at a dose of no more than $60 \mathrm{mg}$ once a week on 5 occasions). Seventy-seven patients were treated with fluorouracil combined with cisplatin chemotherapy (FP group: fluorouracil $4.0 \mathrm{~g} / \mathrm{m}^{2}$, continuous intravenous infusion for $96 \mathrm{hrs}+$ cisplatin $65 \mathrm{mg} /$ $\mathrm{m}^{2}$, intravenous infusion on day 1 , repeated at 3-4 week intervals, 2 courses of treatment); 13 cases received paclitaxel combined with cisplatin chemotherapy (TP group: paclitaxel $135 \mathrm{mg} / \mathrm{m}^{2}$, intravenous infusion on day $1+$ cisplatin $65 \mathrm{mg} / \mathrm{m}^{2}$, intravenous infusion on day 1 , repeated at 3-4 week intervals, 2 courses of treatment).

\section{Statistical methods}

SPSS software, version 19.0 was used for data analysis. The Kaplan-Meier method was used to calculate overall survival (OS) and progress-free survival (PFS). The logrank test was used to compare the difference in survival, and the Cox proportional hazard regression models were used for multivariate analysis.

\section{Follow-up}

At the end of treatment, patients were regularly followed up at the outpatient clinic. In case of clinically suspected recurrence and metastasis, imaging and pathology were obtained.

\section{Results}

\section{Follow-up and survival outcomes}

The deadline for follow-up was February 2017. The median follow-up time was 67 months (range: 8-89 months). 
Table 2 The first recurrence sites in patients

\begin{tabular}{|c|c|c|c|c|}
\hline \multirow[t]{2}{*}{ Factors } & \multirow{2}{*}{$\begin{array}{l}\text { Total } \\
\mathbf{N}\end{array}$} & \multicolumn{3}{|c|}{ Recurrence (N, \%) } \\
\hline & & Local & Distant & Local+Distant \\
\hline \multicolumn{5}{|l|}{ Number of pelvic LNM } \\
\hline $\mathrm{I}-2$ & 97 & $7(7.2)$ & $9(9.3)$ & 0 \\
\hline$\geq 3$ & 58 & $10(17.2)$ & $8(13.8)$ & $\mathrm{I}(\mathrm{I} .7)$ \\
\hline \multicolumn{5}{|l|}{ Number of pelvic LNM sites } \\
\hline $\mathrm{I}-2$ & 125 & $12(9.6)$ & $\mathrm{II}(8.8)$ & 0 \\
\hline$\geq 3$ & 30 & $5(16.7)$ & $6(20)$ & $\mathrm{I}(3.3)$ \\
\hline \multicolumn{5}{|l|}{ Site of pelvic LNM } \\
\hline Common iliac & 28 & $6(21.4)$ & $5(17.9)$ & 0 \\
\hline Common iliac unaffected & 127 & $\mathrm{II}(8.7)$ & $12(9.4)$ & $\mathrm{I}(0.8)$ \\
\hline
\end{tabular}

Abbreviations: LNM, lymph node metastasis.

The follow-up rate was $94.8 \%$ (147 of 155 patients). The 5-year PFS and OS of the entire cohort was $78.1 \%$ and $81.9 \%$, respectively. Table 2 displays the first recurrence noted in patients. Thirty-five patients experienced relapse, $16(16.5 \%)$ had 1 or 2 positive pelvic LNs and $19(32.7 \%)$ had more than 2 positive pelvic LNs; 23 (18.4\%) had 1 or 2 pelvic LNM sites affected and $12(40 \%)$ had more than 2 pelvic LNM sites affected; 11 (39.3\%) had common iliac LNM and 24 (18.9\%) had pelvic LNM other than common iliac LNM. The rate of recurrence was much higher in patients with multiple LNM, multiple LNM sites affected, and those with common iliac LNM.

Thirty-five patients experienced relapse, 17 (48.6\%) with local recurrence (11 pelvic cavity, 6 vaginal stump), and $18(51.4 \%)$ with distant recurrence (2 supraclavicular, 4 para-aortic nodes, 7 lung, 3 bone metastases, and 2 liver). In the radiotherapy group, 15 patients experienced relapse, including 7 patients with local recurrences and 8 patients with distant recurrences. In the concurrent chemoradiotherapy group, 20 patients experienced relapse, including 3 patients with local recurrence and 2 patients with distant metastasis in the DDP group, 4 patients with local recurrence and 7 patients with distant metastasis in the FP group, 3 patients with local recurrence and 1 patient with distant metastasis in the TP group.

\section{The number of pelvic LNM and survival}

Table 3 shows the Log-rank test of clinical and pathological factors. The 5-year OS and PFS of patients with 1 or 2 pelvic LNM was $87.6 \%$ and $83.7 \%$, respectively; for those patients with more than 2 pelvic LNM, it was $72.4 \%$ and $68.4 \%$, respectively. The 5-year PFS and OS of patients with more than 2 pelvic LNM were significantly worse than that of patients with 1 or 2 pelvic $\operatorname{LNM}$ ( $p=0.013$ and $p=0.017$, respectively, Figure 2).

\section{The number of pelvic LNM sites and survival}

The 5-year OS and PFS for patients according to 1 or 2 pelvic LNM sites was $84.8 \%$ and $82.4 \%$, respectively; for patients with more than 2 pelvic LNM sites, the 5-year OS and PFS was 70.0\% and $60.0 \%$, respectively. The 5-year PFS and OS in patients with more than 2 sites affected were significantly poorer than that of patients with 1 or 2 sites affected ( $p=0.008$ and $p=0.045$, respectively, Figure 3 ).

\section{The site of pelvic LNM and survival}

The 5-year OS and PFS for patients with different pelvic LNM sites were as follows: common iliac LNM, 67.9\% and $60.7 \%$, respectively; pelvic LNM other than common iliac LNM, 85.0\% and 81.9\%, respectively. The 5-year PFS and OS for patients with common iliac LNM were significantly poorer than that with pelvic LNM other than common iliac LNM ( $p=0.008, p=0.020$, respectively, Figure 4).

\section{Risk group and survival}

In our study, the survival of patients with any of the following factors was poorer: more than 2 LNM, more than 2 LNM sites, and common iliac LNM. We further analyzed the combined effects of these three risk factors on survival. Five-year OS and PFS were $66.7 \%$ and $55.6 \%$, respectively, in patients with three risk factors; $75.0 \%$ and $68.8 \%$ with two factors; and both $73.3 \%$ and 
Table 3 Log-rank test of clinical and pathological factors

\begin{tabular}{|c|c|c|c|c|}
\hline Factor & $\begin{array}{l}\text { 5y-OS } \\
(\%)\end{array}$ & p-value & $\begin{array}{l}\text { 5y-PFS } \\
(\%)\end{array}$ & p-value \\
\hline Tumor size & & 0.464 & & 0.228 \\
\hline $\mathrm{TI}(\leq 2 \mathrm{~cm})$ & 91.3 & & 91.3 & \\
\hline $\mathrm{T} 2(2<\mathrm{T} \leq 4 \mathrm{~cm})$ & 80.6 & & 79.1 & \\
\hline $\mathrm{T} 3(>4 \mathrm{~cm})$ & 80.0 & & 73.8 & \\
\hline DSI & & 0.942 & & 0.566 \\
\hline$\leq 1 / 2$ & 82.6 & & 82.6 & \\
\hline$>1 / 2$ & 81.8 & & 77.3 & \\
\hline LVSI & & 0.827 & & 0.959 \\
\hline Negative & 80.6 & & 77.8 & \\
\hline Positive & 82.4 & & 78.2 & \\
\hline Surgical margin & & 0.525 & & 0.765 \\
\hline Negative & 82.4 & & 78.4 & \\
\hline Positive & 71.4 & & 71.4 & \\
\hline Number of pelvic & & 0.017 & & 0.013 \\
\hline LNM & & & & \\
\hline $\mathrm{I}-2$ & 87.6 & & 83.7 & \\
\hline$\geq 3$ & 72.4 & & 68.4 & \\
\hline Site of pelvic LNM & & 0.020 & & 0.008 \\
\hline Common iliac & 67.9 & & 60.7 & \\
\hline Common iliac & 85.0 & & 81.9 & \\
\hline unaffected & & & & \\
\hline Number of pelvic & & 0.045 & & 0.008 \\
\hline LNM sites & & & & \\
\hline $\mathrm{I}-2$ & 84.8 & & 82.4 & \\
\hline$\geq 3$ & 70.0 & & 60.0 & \\
\hline PI & & 0.517 & & 0.871 \\
\hline Negative & 81.4 & & 77.9 & \\
\hline Positive & 90.0 & & 80.0 & \\
\hline No. of resected & & 0.736 & & 0.736 \\
\hline nodes & & & & \\
\hline$\leq 18$ & 80.4 & & 76.1 & \\
\hline$>18$ & 82.6 & & 78.9 & \\
\hline Type of & & 0.859 & & 0.919 \\
\hline lymphadenectomy & & & & \\
\hline Pelvic only & 81.4 & & 77.9 & \\
\hline Pelvic + para-aortic & 83.3 & & 78.6 & \\
\hline
\end{tabular}

Abbreviations: DSI, depth of stromal invasion; LVSI, lymphovascular space invasion; LNM, lymph node metastasis; OS, overall survival; PI, parametrial invasion; PFS, progress-free survival.

$73.3 \%$ with one risk factor; and $89.0 \%$ and $85.7 \%$ without any of the aforementioned risk factors. The patients were combined into three groups according to survival: group A had good survival (without risk factors), group B for medium survival (with one or two risk factors), and group $\mathrm{C}$ for poor survival (with all three risk factors). Figure 5 shows OS and PFS curves for the three groups.

\section{Discussion}

Before the 2018 FIGO staging system, the stages of cervical cancer were clinically staged according to physical examination and did not consider the status of LN. However, LNM is a significant predictor of increasing recurrence and metastasis of cervical cancer. Several retrospective studies have reported the 5-year OS is between $80 \%$ and $100 \%$ in those patients with stage IB-IIB disease without LNM. However, in those with LNM, the 5-year OS dropped to between $47 \%$ and $78 \% .{ }^{10-13}$ The FIGO Committee revised the staging system for cervical cancer in 2018. ${ }^{1}$ One of the changes made was the addition of stage IIIC1, referring to pelvic LNM only. However, different LNM status significantly affects prognosis. Compared with LNM, the number of pelvic LNM has a greater impact on prognosis. Research by Uno et $\mathrm{al}^{5}$ showed that the 5-year OS of patients with negative pelvic LN and one positive pelvic LN were $89 \%$ and $83 \%$, respectively. However, when the number of positive pelvic LN exceeded two, the 5-year OS significantly dropped to $58 \%$. The study by Ditto also indicated that the OS of the patients concomitantly declined with increasing positive nodes. ${ }^{14}$ Takeda et $\mathrm{al}^{4}$ reported that the patients with $\geq 3$ positive pelvic $\mathrm{LN}$ had a poor prognosis, where the 5-year OS was $20.2 \%$ in those with stage IB-IIB disease. In our study, the 5-year PFS and OS of patients with more than 2 pelvic LNM was significantly lower than that of patients with 1 or 2 pelvic LNM $(68.4 \%$ vs $83.7 \%, p=0.013 ; 72.4 \%$ vs $87.6 \%$, $p=0.017$, respectively). However, the survival rate in our study was better than that observed in previous studies, probably because our study included 2009 FIGO IB-IIA patients, and did not include IIB patients.

In addition to the number of pelvic LNM, several studies have assessed the impact of the site of LNM in cervical cancer survival. In the study by Sakuragi et $\mathrm{al}^{3}{ }^{3}$ the 5 -year OS of patients with more than 2 pelvic LNM sites was significantly lower than those with 1 or 2 pelvic LNM sites ( $26.5 \%$ vs $84.9 \%$ ), while the survival rate between patients with one pelvic LNM site and with non-positive nodes was not distinctly different. Other studies have shown that the increase in the number of LNM site reduced the 5-year OS by $50-60 \%$ and that patients with more than one LNM site had a distinctly poorer survival compared with those with one site. $^{4,15}$ The outcome of our study correlated with those reported in the literature. The 5-year PFS and OS of patients 
A

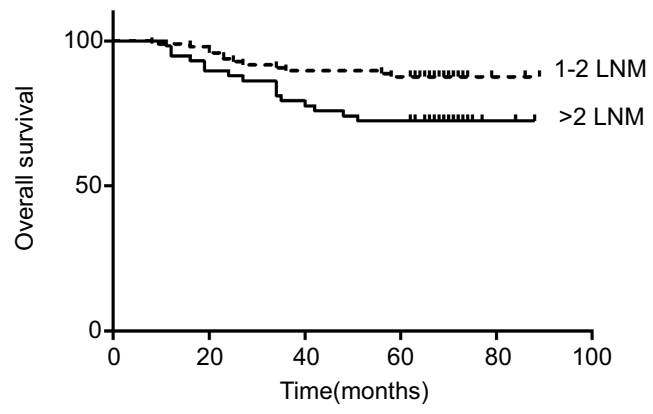

B

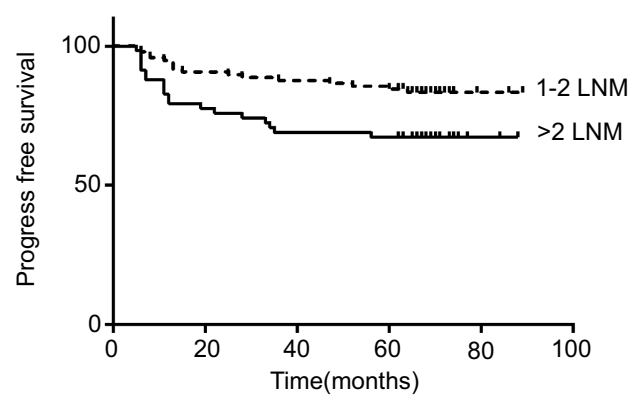

Figure 2 OS and PFS curves for number of LNM.

Abbreviations: OS, overall survival; PFS, progress-free survival; LNM, lymph node metastasis.

A

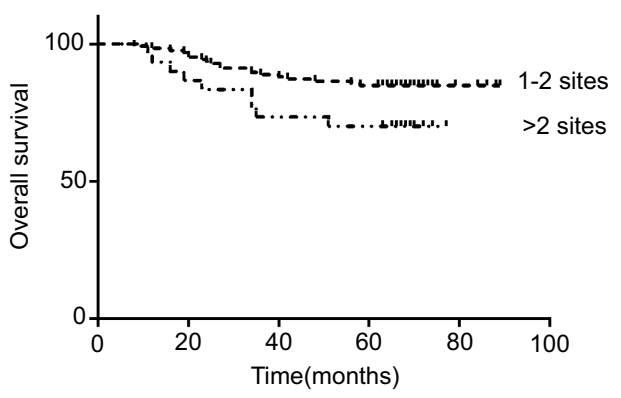

B

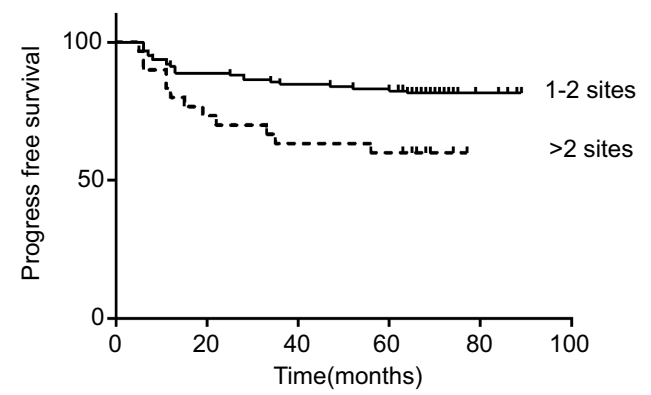

Figure 3 OS and PFS curves for number of LNM sites.

Abbreviations: OS, overall survival; PFS, progress-free survival; LNM, lymph node metastasis.

A

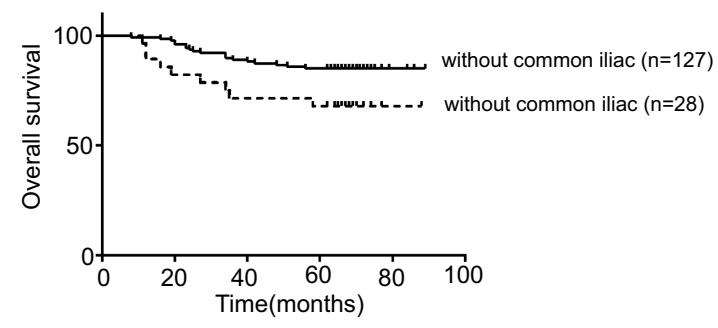

B

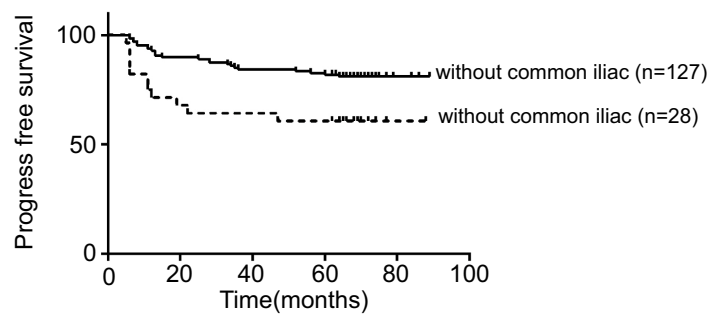

Figure 4 OS and PFS curves for LNM site.

Abbreviations: OS, overall survival; PFS, progress-free survival; LNM, lymph node metastasis.

with more than 2 pelvic LNM sites were poorer than in patients with 1 or 2 pelvic LNM sites.

In pelvic LNM of cervical cancer, the incidence of common iliac LNM was lower. However, patients with common iliac LNM always had a lower survival rate. The 5-year OS of patients with common iliac LNM was between $25 \%$ and $47.8 \%{ }^{8,16,17}$ Common iliac LNM is considered to be one of the prognostic factors associated with poorer survival. In the study by Huang et al, the 5-year OS of patients with non-pelvic LNM, pelvic LNM without common iliac LNM, and common iliac LNM were $91.5 \%, 67.5 \%$, and $46.1 \%$, respectively. ${ }^{18}$ In another research, in comparison with the patients with pelvic LNM other than common iliac LNM, the prognosis of the patients with common iliac LNM was poorer. The 5-year OS of patients with common iliac LNM was $58.3 \%{ }^{19}$ In this study, although the 5-year survival rate of patients with common iliac LNM was slightly higher than that reported in the previous literature, it was significantly lower than in those without common iliac LNM. 

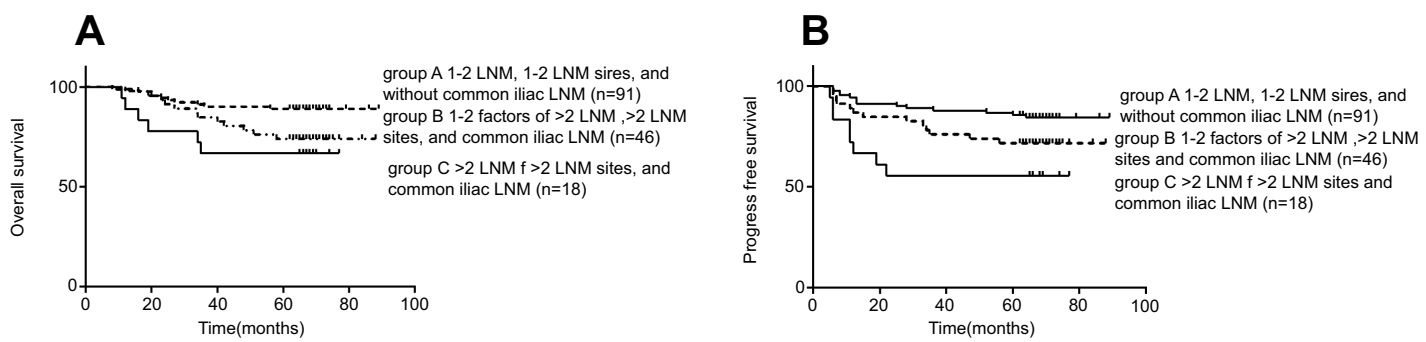

Figure 5 OS and PFS curves for different combinations of risk factors. (A) The 5 -year OS of group A, group B, and group $C$ were $89.0 \%, 73.9 \%$, and $66.7 \%$, respectively. The survival curves of group $B$ and group $C$ were significantly worse than the survival of group $A(p<0.05)$ (group $A$ vs group $B, p=0.027$; group $A$ vs group $C$, $p=0.008$; group $B$ vs group $C, p=0.432$ ). (B) The 5 -year PFS of group $A$, group $B$, and group $C$ were $85.7 \%, 71.7 \%$, and $55.6 \%$, respectively. The survival curve of group $C$ was significantly worse than the survival curve of group $A(p<0.05)$ (group $A$ vs group $B, p=0.067$; group $A$ vs group $C, p=0.001$; group $B$ vs group $C$, $p=0.139$ ).

Abbreviations: LNM, lymph node metastasis; OS, overall survival; PFS, progress-free survival.

In our study, the survival of patients with any of the following factors was poorer: more than $2 \mathrm{LNM}$, more than 2 LNM sites, or common iliac LNM. We further analyzed the combined effects of three risk factors (the number of LNM, the number of LNM sites, and LNM site) on prognosis. The survival of patients was poorer in those with at least one of the following factors: more than $2 \mathrm{LNM}$, more than $2 \mathrm{LNM}$ sites, and common iliac LNM. Compared with other patients, the survival of patients with these three factors was the worst. The survival for stage IIIC1p cervical cancer varies with the factors of pelvic metastasis LNs, that is to say, stage IIIC1p is heterogeneous. Therefore, we recommend that the combined evaluation of the factors of positive pelvic LNs is a more comprehensive and reasonable approach in evaluating the prognosis of cervical cancer. In clinical practice, stratified treatment should be adopted according to different prognoses. More appropriate treatment strategies should be selected in order to improve the survival rate of patients with the aforementioned risk factors. One possible treatment is to use cisplatin-based doublet chemotherapy during radiotherapy. Recently, a meta-analysis suggested that platinumbased doublet chemoradiotherapy could improve the OS and PFS of patients compared with concurrent chemoradiotherapy with weekly cisplatin. ${ }^{20}$ Another possible treatment was the addition of consolidation chemotherapy after adjuvant chemoradiotherapy. In Mabuchi's research, paclitaxel and carboplatin-based consolidation chemotherapy was highly effective in early stage cervical cancer patients with positive lymph nodes after surgery. ${ }^{21}$ Currently, the GOG0724 clinical trial has been running since 2009 , which evaluates the efficacy of consolidation chemotherapy with paclitaxel and carboplatin in patients with high-risk factors after surgery. The results of this trial are eagerly awaited.

At present, 2018 FIGO IIIC1p stage refers to pelvic LNM only, without considering the different variables associated with LNM such as number of LNM, number of LNM sites, and specific LNM site. Currently, there is no stratification of treatment recommendations for patients. Therefore, we recommend that the FIGO Committee should take these factors into account when revising the staging system in the future. Our study has the following shortcomings. First, there are inherent limitations of retrospective research. Second, the cohort number was quite small. Moreover, this study is a single-center study.

In conclusion, our research shows that more than 2 LNM, more than 2 LNM sites, and common iliac LNM were risk factors for poor survival in stage IIIC1p cervical cancer patients. The survival of stage IIIC1p cervical cancer varies with an increasing number of risk factors. The combined evaluation of the factors of positive pelvic LNs is a more comprehensive and reasonable approach in evaluating the prognosis of cervical cancer. Patients with one or more of the aforementioned factors should be appropriately treated in order to improve the survival rate, especially those with all three factors. It is hoped that prospective clinical studies with a large sample size for clinical validation of stage III C1p will provide a basis for future revision of the staging system.

\section{Abbreviation list}

LNs, lymph nodes; LNM, lymph node metastasis; OS, overall survival; PFS, progression-free survival.

\section{Acknowledgments}

This study was supported by the Zhejiang Province Health Department Foundation. (No.2016KYA036)

\section{Disclosure}

The authors report no conflicts of interest in this work. 


\section{References}

1. Bhatla N, Aoki D, Sharma DN, Sankaranarayanan R. Cancer of the cervix uteri. Int $J$ Gynaecol Obstet. 2018;143(Suppl 2):22-36. doi:10.1002/ijgo.12611

2. Matsuo K, Machida H, Mandelbaum RS, Konishi I, Mikami M. Validation of the 2018 FIGO cervical cancer staging system. Gynecol Oncol. 2019;152(1):87-93. doi:10.1016/j. ygyno.2018.10.026

3. Sakuragi N, Satoh C, Takeda N, et al. Incidence and distribution pattern of pelvic and paraaortic lymph node metastasis in patients with stages IB, IIA, and IIB cervical carcinoma treated with radical hysterectomy. Cancer. 1999;85(7):1547-1554. doi:10.1002/(SICI) 1097-0142(19990401)85:7<1547::AID-CNCR16>3.0.CO;2-2

4. Takeda N, Sakuragi N, Takeda M, et al. Multivariate analysis of histopathologic prognostic factors for invasive cervical cancer treated with radical hysterectomy and systematic retroperitoneal lymphadenectomy. Acta Obstet Gynecol Scand. 2002;81(12):1144-1151.

5. Uno T, Ito H, Itami J, et al. Postoperative radiation therapy for stage IB-IIB carcinoma of the cervix with poor prognostic factors. Anticancer Res. 2000;20(3B):2235-2239.

6. Lee YJ, Kim DY, Lee SW, et al. A postoperative scoring system for distant recurrence in node-positive cervical cancer patients after radical hysterectomy and pelvic lymph node dissection with para-aortic lymph node sampling or dissection. Gynecol Oncol. 2017;144(3):536-540. doi:10.1016/j.ygyno.2017.01.001

7. Kwon J, Eom KY, Kim YS, et al. The prognostic impact of the number of metastatic lymph nodes and a new prognostic scoring system for recurrence in early-stage cervical cancer with high risk factors: a multicenter cohort study (KROG 15-04). Cancer Res Treat. 2018;50(3):964-974. doi:10.4143/crt.2017.346

8. Aoki Y, Sasaki M, Watanabe $M$, et al. High-risk group in node-positive patients with stage IB, IIA, and IIB cervical carcinoma after radical hysterectomy and postoperative pelvic irradiation. Gynecol Oncol. 2000;77(2):305-309. doi:10.1006/gyno.2000.5788

9. Chen Z, Huang K, Lu Z, et al. Risk model in stage IB1-IIB cervical cancer with positive node after radical hysterectomy. Onco Targets Ther. 2016;9:3171-3179. doi:10.2147/OTT.S94151

10. Havrilesky LJ, Leath CA, Huh W, et al. Radical hysterectomy and pelvic lymphadenectomy for stage IB2 cervical cancer. Gynecol Oncol. 2004;93(2):429-434. doi:10.1016/j.ygyno.2004.01.038

11. Morice P, Castaigne D, Pautier P, et al. Interest of pelvic and paraaortic lymphadenectomy in patients with stage IB and II cervical carcinoma. Gynecol Oncol. 1999;73(1):106-110. doi:10.1006/gyno.1998.5308
12. Kim SM, Choi HS, Byun JS. Overall 5-year survival rate and prognostic factors in patients with stage IB and IIA cervical cancer treated by radical hysterectomy and pelvic lymph node dissection. Int J Gynecol Cancer. 2000;10(4):305-312. doi:10.1046/j.1525-1438.2000.010004305.x

13. Lai CH, Hong JH, Hsueh S, et al. Preoperative prognostic variables and the impact of postoperative adjuvant therapy on the outcomes of stage IB or II cervical carcinoma patients with or without pelvic lymph node metastases: an analysis of 891 cases. Cancer. 1999;85(7):1537-1546.

14. Ditto A, Martinelli F, Lo Vullo S, et al. The role of lymphadenectomy in cervical cancer patients: the significance of the number and the status of lymph nodes removed in 526 cases treated in a single institution. Ann Surg Oncol. 2013;20(12):3948-3954. doi:10.1245/ s10434-013-3067-6

15. Inoue $\mathrm{T}$, Morita $\mathrm{K}$. The prognostic significance of number of positive nodes in cervical carcinoma stages IB, IIA, and IIB. Cancer. 1990;65 (9):1923-1927.

16. Yeh SA, Wan Leung S, Wang CJ, Chen HC. Postoperative radiotherapy in early stage carcinoma of the uterine cervix: treatment results and prognostic factors. Gynecol Oncol. 1999;72(1):10-15. doi:10.1006/gyno.1998.5217

17. Kodaira T, Fuwa N, Nakanishi T, et al. Long-term clinical outcomes of postoperative pelvic radiotherapy with or without prophylactic paraaortic irradiation for stage I-II cervical carcinoma with positive lymph nodes: retrospective analysis of predictive variables regarding survival and failure patterns. Am J Clin Oncol. 2004;27(2):140-148.

18. Huang L, Zheng M, Liu JH, et al. Risk factors and prognosis of IB-IIB cervical carcinoma with common iliac lymph node metastasis. Chin J Cancer. 2010;29(4):431-435.

19. Tsuruga T, Fujimoto A, Kawana K, et al. Radical hysterectomy with or without para-aortic lymphadenectomy for patients with stage IB2, IIA2, and IIB cervical cancer: outcomes for a series of 308 patients. Int J Clin Oncol. 2016;21(2):359-366. doi:10.1007/ s10147-015-0907-3

20. Petrelli F, De Stefani A, Raspagliesi F, Lorusso D, Barni S. Radiotherapy with concurrent cisplatin-based doublet or weekly cisplatin for cervical cancer: a systematic review and meta-analysis. Gynecol Oncol. 2014;134(1):166-171. doi:10.1016/j.ygyno.20 14.04.049

21. Mabuchi S, Isohashi F, Yokoi T, et al. A phase II study of postoperative concurrent carboplatin and paclitaxel combined with intensity-modulated pelvic radiotherapy followed by consolidation chemotherapy in surgically treated cervical cancer patients with positive pelvic lymph nodes. Gynecol Oncol. 2016;141(2):240-246. doi:10.1016/j.ygyno.2016.02.011

\section{Publish your work in this journal}

Cancer Management and Research is an international, peer-reviewed open access journal focusing on cancer research and the optimal use of preventative and integrated treatment interventions to achieve improved outcomes, enhanced survival and quality of life for the cancer patient.
The manuscript management system is completely online and includes a very quick and fair peer-review system, which is all easy to use. Visit http://www.dovepress.com/testimonials.php to read real quotes from published authors. 